ESJ Social Sciences

\title{
Décentralisation des politiques éducatives en Côte d'Ivoire : Les effets structurants des motivations politiques sur les résultats de l'action publique locale
}

\author{
Pregnon Claude Nahi \\ Enseignant-Chercheur, \\ Université Peleforo Gon Coulibaly de Korhogo, Côte d'Ivoire
}

$\underline{\text { Doi:10.19044/esj.2021.v17n1p167 }}$

Submitted: 04 November 2020

Accepted: 16 December 2020

Published: 31 January 2021
Copyright 2021 Author(s)

Under Creative Commons BY-NC-ND

4.0 OPEN ACCESS

Cite As:

Nahi C.P. (2021). Décentralisation des politiques éducatives en Côte d'Ivoire : Les effets structurants des motivations politiques sur les résultats de l'action publique locale. European Scientific Journal, ESJ, 17(1), 167. https://doi.org/10.19044/esj.2021.v17n1p167

\section{Résumé}

La décentralisation est apparue vers les années 80 en Côte d'Ivoire au moment de la diminution des ressources de l'État et des restrictions budgétaires imposées par le FMI. Au nombre des solutions envisagées pour juguler la conjoncture économique, il a été envisagé le transfert d'importantes prérogatives de l'Etat central vers les collectivités territoriales parmi lesquelles figurent le service public de l'enseignement. Si la décentralisation des politiques éducatives a été conçue à l'origine pour contribuer à l'efficacité de l'action publique en la rapprochant du citoyen, ses résultats semblent de plus en plus mitigés. Pour rendre compte de l'inefficacité du processus, la crise économique a été majoritairement mobilisée comme principal facteur explicatif. Bien que n'éludant pas la pertinence et la valeur heuristique de cet argument, ce travail s'intéresse quant à lui aux logiques propres des acteurs, piste qui semble n'avoir pas été suffisamment explorée. En d'autres termes, l'étude soutient que les politiques éducatives locales sont inefficaces parce qu'elles sont structurées par les intérêts matériels et positionnels des décideurs liés aux enjeux électoraux.

Mots clés : Crise économique, décentralisation, service public de l'enseignement, agenda, processus décisionnel, motivations électoralistes 


\title{
Decentralisation of Education Policies in Côte d'Ivoire: The Structuring Effects of Political Motivations on the Results of Public Action
}

\author{
Pregnon Claude Nahi \\ Enseignant-Chercheur, \\ Université Peleforo Gon Coulibaly de Korhogo, Côte d'Ivoire
}

\begin{abstract}
The decentralization emerged in the 1980s in Côte d'Ivoire at a time of declining state resources and budgetary restrictions imposed by the IMF. Among the solutions envisaged to curb the economic situation, the transfer of important prerogatives from the central state to local authorities, including the public education service, was envisaged. Although the decentralisation of education policies was originally designed to contribute to the effectiveness of public action by bringing it closer to the citizen, its results seem increasingly mixed. To account for the inefficiency of the process, the economic crisis has been mobilized as the main explanatory factor. While not evading the relevance and the heuristic value of this argument, this work focuses on the actors' own logics, an avenue that seems to have been insufficiently explored. In other words, the study argues that local education policies are ineffective because they are structured by the material and positional interests of decisionmakers linked to electoral issues.
\end{abstract}

Keywords: Economic crisis, decentralization, public service education, agenda, decision-making process, electoral motivation

\section{Introduction}

Le modèle de l'État centralisé qui s'est longtemps imposé en Côted'Ivoire, repose d'abord sur l'idée selon laquelle, l'État est seul à même de définir l'intérêt général et d'arbitrer entre celui-ci et les intérêts particuliers (Wodie, 1996). L'État se voit reconnaître un rôle exclusif pour structurer et coordonner les activités de la société. C'est pourquoi, dès l'indépendance du pays en 1960, les pouvoirs publics ivoiriens ont placé l'éducation nationale au rang des priorités en affichant leur volonté politique d'atteindre un taux de scolarisation de $100 \%$ (Proteau, 2002). Pour ce faire, ils ont réservé chaque année, plus de $40 \%$ du budget national à l'enseignement (Hugon, Vimard, \& Tapinos, 2003).

Le service public d'éducation et d'enseignement a donc été pensé comme devant être le véhicule majeur de dissémination des valeurs de la 
république une et indivisible en renforçant les instruments d'une bonne éducation à la citoyenneté et à l'esprit républicain ${ }^{1}$. L'État reste garant du bon fonctionnement du service public national et maitre du contenu des programmes, de l'organisation des examens, du contrôle pédagogique, du recrutement et de la nomination des personnels. Cependant, le système éducatif ivoirien a été progressivement confronté à une longue crise (Proteau, 2002) économique et structurelle ${ }^{2}$ consécutive aux faiblesses constatées au niveau de la planification (Semiti, 2006), de la programmation et de l'évaluation. Ainsi, pour comprendre la trajectoire qu'a suivi le système éducatif ivoirien en termes de continuité ou des rupture, la périodisation suivante, peut être avancée : «Dans une première période, de l'indépendance à la fin des années 1980, l'État a été le principal acteur d'une planification scolaire financée sur les fonds de la coopération bilatérale et destinée, au moins en théorie, à étendre et à élargir la scolarisation en opposition aux politiques éducatives coloniales restrictives; dans une seconde période, de la fin des années 1980 à nos jours, (...), les bailleurs de fonds internationaux conditionnent l'aide financière à une restructuration du système éducatif, limitant ainsi l'autonomie d'intervention de l'État dans le domaine scolaire du fait de l'imposition de nouvelles normes (...) » (Lanoue, 2004, p. 85), .

Pour pallier aux effets de cette crise et respecter les exigences des nouvelles conditionnalités des bailleurs, l'État de Côte-d'Ivoire, a inscrit sur son agenda la politique de décentralisation des politiques éducatives. On peut appréhender la décentralisation en éducation comme: «le transfert de responsabilités, en matière de planification, management, financement et allocation des ressources, du gouvernement central vers des unités locales subnationales publiques ou privées, ces unités pouvant soit être directement placées sous l'autorité du gouvernement, soit disposer d'une autonomie partielle ou totale (Mons, 2004, p. 42) ». Les motivations de la décentralisation des politiques éducatives à l'instar de celles initiées ailleurs et dans des contextes différents peuvent se résumer en trois catégories (Rey, 2013). Primo, elles le sont, pour des raisons politiques en vue d'instaurer une gouvernance participative qui associe les citoyens à leur école. Secundo, c'est pour des finalités économiques en vue d'atteindre une plus grande efficience économique et sociale et tertio pour améliorer l'efficacité des enseignements

1 À cet effet, l'article premier de la loi $\mathrm{n}^{\circ}$ 95-696 du 7 septembre 1995 relative à l'Enseignement dispose que : «Le droit à l'éducation est garanti à chaque citoyen afin de lui permettre d'acquérir le savoir, de développer sa personnalité, d'élever son niveau de formation, de s'insérer dans la vie sociale, culturelle et professionnelle et d'exercer sa citoyenneté ».

${ }^{2}$ La planification n'a pas toujours été respectée en matière de création d'écoles en Côte d'Ivoire de sorte que les estimations correctes des besoins d'éducation, surtout en ce qui se rapporte aux ressources humaines, n'ont pas été effectuées. Les possibilités budgétaires de l'État n'ont pas également été suffisamment prises en compte dans les programmes éducatifs. 
en les adaptant aux besoins des élèves. Cependant, s'il est indéniable que la décentralisation des politiques éducatives présente des avantages certains, des retours d'expériences peu positifs commandent de relativiser leur efficacité.

En effet, en Afrique (Marie \& Idelman, 2010), de nombreux pays ont opté pour une décentralisation minimale. Cette option qui est caractérisée par un État (Gacha, 2009) généralement concepteur, opérateur et contrôleur du système éducatif se matérialise par des transferts restreints et un processus décisionnel stato-centré. Pourtant, une telle option n'est pas toujours sans conséquence sur le rendement de l'action publique locale. C'est-à-dire que : « là où la supervision et l'appui de l'État sont faibles et où son absence n'est pas compensée par une obligation locale forte de rendre compte, l'inefficacité et la mauvaise gestion qui ont caractérisé la gestion centrale peuvent se reproduire, sinon se multiplier, aux niveaux inférieurs » (Lugaz et al., s. d., p. 140). En effet, si des pays aux caractéristiques très différentes ont adopté dans leur politique pour cette réforme ambitieuse et complexe : «il y a des doutes, cependant, quant à ses objectifs et son impact » (Lugaz et al., s. d., p. 7). En d'autres termes, la décentralisation peut contenir des «dangers » (Prud'homme, 1995) qui peuvent mettre en doute l'idée qu'elle puisse conduire à une allocation plus efficace des ressources. Ce qui explique d'ailleurs que la politique de décentralisation en Côte-d'Ivoire n'ait pas toujours pu atteindre les objectifs ${ }^{3}$ qui lui ont été assignés. Autrement dit : « la politique de décentralisation actuelle connaît certes des avancées considérables au niveau administratif, politique, institutionnel, territorial et économique, mais reste à un stade embryonnaire en ce qui concerne les réalisations et les transferts de compétences » (Soumahoro, 2015, p. 35). C'est à dire qu'à l'épreuve du terrain, l'on observe qu'il y a un décalage entre l'action publique locale et les aspirations des populations (Adomon, 2015). Les causes de l'inefficacité des politiques éducatives décentralisées ont été diversement abordées. Pour apporter une réponse à cette préoccupation, de nombreuses études ont tenté d'établir une corrélation entre la crise économique (Mel, 2015) et l'ineffectivité (Atta, 2001) des compétences transférées en Côte d'Ivoire. Or, une réflexion sur la décentralisation est indissociable d'une réflexion sur l'organisation des pouvoirs (Dramé, 1998) dans la mesure où elle vient rompre avec le modèle de l'État centralisé, la décentralisation implique une nouvelle distribution des pouvoirs.

C'est pourquoi, la présente étude, veut mettre en exergue un aspect important de l'élucidation de cette problématique qui n'a pas été suffisamment pris en compte par les études antérieures. Elle veut mettre l'accent sur le mode de gestion des deniers publics marqué par la non-transparence (Blundo, 2001),

3 Voir les actes des états généraux de la table ronde de la Décentralisation et du Développement Local. Ministère de l'Administration du Territoire, 2007. 
les pratiques clientélistes des élus locaux et son corollaire de mise en œuvre des projets locaux biaisé par des motivations essentiellement électoralistes (Douillet \& Robert, 2007). Le poids des motivations politiques peut constituer un obstacle majeur à l'efficacité des politiques décentralisées lorsque dans la prise en charge des compétences transférées, les intérêts personnels et politiques de l'ordre politique prévalent sur ceux des populations locales. Plus précisément, les alternances électorales peuvent avoir un double impact : Elles peuvent agir sur la conduite des politiques et sur la façon dont les protagonistes des politiques publiques modifient leur implication en fonction des échéances électorales à venir. Ce travail est essentiellement basé sur la théorie économique de la démocratie développée par Anthony Downs (Downs, 2013), qui part de l'idée que la motivation principale des élus consiste à profiter des avantages personnels que peuvent leur conférer les fonctions publiques en termes de revenu, de prestige et de pouvoir. Autrement dit, c'est la recherche du succès électoral qui explique pourquoi des élus vont s'engager pour la mise en place de politiques publiques. L'approche conceptuelle basée sur les idées, des intérêts et des institutions « Trois I » (Palier \& Surel, 2005) a contribué à opérationnaliser l'orientation théorique. L'enquête et la collecte des données de terrain se sont déroulées dans la commune de Cocody située dans le district d'Abidjan.

\section{La politisation des politiques éducatives locales}

Le transfert des pouvoirs de gestion et de décision à des acteurs plus proches des réalités où s'expriment les besoins des communautés est le principe fondamental qui gouverne toute politique de décentralisation. C'est dans cette optique que l'État ivoirien a adopté pour le principe du transfert de certaines compétences aux collectivités territoriales en matière d'éducation ${ }^{4}$. Cependant, le choix de la décentralisation minimale fait que l'État demeure encore la pièce maitresse de ces politiques ${ }^{5}$. En effet, il apparait que, dans l'exercice des compétences transférées, les motivations politiques et électoralistes (Maillard, s. d.), structurent significativement les politiques éducatives locales. D'une part, le gouvernement central semble réticent à partager ses prérogatives avec les pouvoirs locaux et rechigne dans bien des cas, à décentraliser certaines prérogatives aux structures régionales et locales.

D'autre part, ce manque de volonté politique entraine une insuffisance de l'encadrement juridique du processus de transfert des compétences qui finit par poser problème dans l'appropriation des compétences transférées par les

\footnotetext{
${ }^{4}$ Ce transfert de compétence s'opère entre autre par la loi n ${ }^{\circ} 2003-208$ du 7 juillet 2003 portant transfert et répartition de compétences de l'État aux Collectivités territoriales.

${ }^{5}$ Loi n ${ }^{\circ} 95-696$ du 07 Septembre 1995 relative à l'Enseignement stipule en son article premier que : «L'éducation est l'une des priorités de l'État. Elle constitue le service public de l'Enseignement».
} 
acteurs locaux en ce qui concerne leur compréhension des textes (Ministère de l'Administration du Territoire a organisé les \& États Généraux de la Décentralisation et du Développement Local, 2007). In fine, cette absence de clarté favorise une instrumentalisation des compétences transférées à des fins électoralistes par les élus locaux.

\section{Une autonomie réduite par un cadre juridique contraignant}

D'une manière générale, la décentralisation se définit comme le processus par lequel le pouvoir central transfère certaines de ses prérogatives au profit de structures locales. Il s'agit, en d'autres termes, d'une technique de gestion administrative par laquelle l'État transfère des postes d'autorité, des compétences et des capacités à des organes relativement autonomes (Greffe, 2005). Ainsi, la décentralisation peut être assimilée à des situations, des modèles et des processus variés en fonction des motivations politiques de chaque pays. En Côte d'Ivoire, la décentralisation n'apparait que vers les années 80 à la faveur de la crise économique (Adiko, 2003). En fait, la découverte de l'importance des acteurs à l'échelle locale n'intervient qu'au moment de la diminution des ressources de l'État, du problème du règlement de la dette extérieure et des restrictions budgétaires imposées par le FMI (Atta \& Gobe, 2013). La décentralisation territoriale (Gacha, 2009) qui se rapporte à l'étude, bien qu'ayant été prévu par l'article 68 de la constitution du 3 novembre 1960, n'est réellement intervenue que vingt ans après ${ }^{6}$. C'est-à-dire qu'il faut attendre, les lois $\mathrm{n}^{\circ} 80-1180$ sur l'organisation municipale, $\mathrm{n}^{\circ} 80$ 1181 sur le régime électoral, $\mathrm{n}^{\circ}$ 80-1182 sur la création de la ville d'Abidjan du 17 octobre 1980 pour assister à la phase de démarrage effectif de l'opération de communalisation avec l'organisation, à l'échelle nationale, des premières élections municipales en 1980.

Depuis cette date, le processus a évolué avec la création de régions décentralisées ${ }^{7}$ et de districts ${ }^{8}$ autonomes. Dans la forme, le fonctionnement et l'organisation de l'administration instituée ont été soumis à une double subordination : juridique et politique (Mambo, 2009). Cependant, en Côte d'Ivoire, la mise en œuvre de la bureaucratie n'a pas nécessairement obéi aux principes de l'idéal type Wébérien. Par conséquent, le procédé technique utilisé pour l'organisation de l'administration a généralement pris une forme

\footnotetext{
${ }^{6}$ Cette décentralisation a connu deux principaux moments : la phase d'expérimentation et celle de consolidation. La phase d'expérimentation débute avec la loi de la loi n ${ }^{\circ} 78-07 \mathrm{du} 09$ janvier 1978 portant création, sous un régime unique, de 26 nouvelles communes de plein exercice.

${ }^{7}$ Le pays compte aujourd'hui trente et une (31) collectivités territoriales régionales.

${ }^{8}$ La loi découpe formellement le territoire national en douze (12) Districts, circonscriptions administratives, et deux (02) Districts Autonomes que sont Abidjan et Yamoussoukro. Seuls les deux districts autonomes sont présentement fonctionnels.
} 
plus «autoritaire $»^{9}$. En ce qui concerne le second aspect, c'est-à-dire la subordination politique, elle s'observe à travers le cadre juridique et le mode d'organisation.

Dans le premier cas, au plan juridique, le critère principal de l'État a été celui de l'exercice de la souveraineté, c'est-à-dire un pouvoir inconditionné faisant du pouvoir central, la source principale de décisions, dont tous les autres pouvoirs dérivent. Or, en Côte d'Ivoire, comme dans de nombreux africains, l'État, le politique, le peuple sont intentionnellement indifférenciés. De sorte que, le principe hiérarchique qui est la clef de voûte et l'élément central de l'organisation bureaucratique en ce sens qu'il permet de garantir la subordination et la soumission de l'administration d'État aux orientations souhaitées ${ }^{10}$ par les autorités politiques légitimes n'a servi qu'à accentuer la centralisation. Autrement dit, contrairement au schéma classique de subordination politique, en Côte d'Ivoire : «La transplantation de l'administration publique française dans les États d'Afrique noire francophone en général et en Côte d'Ivoire en particulier s'est faite de manière imparfaite, la dose d'autonomie ayant été complétement vidée ou presque. L'administration est presqu'entièrement subordonnée aux organes politiques, ne bénéficiant que d'une autonomie particulièrement faible » (Degni Segui Rene, 2013, p. 21). Ce processus est le résultat de «hyperprésidentialisme » (Nahi, 2018) que les différentes constitutions ivoiriennes ont consacré. Une sorte de «dictature constitutionnelle» qui concentre tous les pouvoirs aux mains du chef de l'État et de l'Exécutif en sa qualité de chef de l'administration. Ce qui fait qu'au niveau des collectivités territoriales, les gouvernements successifs ivoiriens même quand ils décentralisent les fonctions, ils retiennent souvent à leur niveau, les rôles politiques les plus importants et les rôles de supervision. Or, dans les rapports entre les collectivités décentralisées et le pouvoir central, il est constant que le rapport n'est pas d'ordre hiérarchique et qu'il devrait se réduire à une simple surveillance ou supervision (Caillosse, 1988). À l'opposé d'une telle vision, les procédés de contrôle utilisés en Côte d'Ivoire, sont plutôt de nature à affaiblir l'autonomie des collectivités et à renforcer leur dépendance vis-à-vis de l'exécutif. Dans les faits, nous sommes passés d'un contrôle politique plus subtile avec « le contrôle d'opportunité » et celui de la « tutelle-conseil ${ }^{11}$ qui faisait passer la collectivité locale « comme une majeure non émancipée a qui l'État doit donner : assistance, conseil et soutien » (Dégni-Ségui, 2013, p. 99) à une véritable subordination politique. En d'autres termes, le législateur

\footnotetext{
${ }^{9}$ L'expression est du professeur Degni Segui.

${ }^{10}$ La hiérarchie permet de réaliser l'unité de commandèrent au profit du sommet, dont les ordres sont répercutés et exécutés fidèlement en suivant les lignes de la hiérarchie.

${ }^{11}$ La tutelle conseil est un ajout du législateur ivoirien à la tutelle classique héritée du droit français.
} 
ivoirien ${ }^{12}$ a désormais fait du contrôle a priori la règle et le contrôle a posteriori l'exception au terme de l'article 44 de la loi sur l'orientation de l'organisation générale de l'administration de 2014. Avec cette nouvelle loi, nous sommes en en face d'une forme déguisée de contrôle hiérarchique qui soumet les collectivités territoriales a une subordination politique.

En fin de compte, si la décentralisation vise formellement une gestion autonome des compétences transférées aux collectivités, il apparait clairement qu'au vu du cadre institutionnel et juridique, qu'il subsiste encore une méfiance du pouvoir central. Et cette méfiance se révèle à travers le cadre juridique et institutionnel des politiques éducatives locales. En Côte d'Ivoire, la décentralisation des politiques éducatives est prévue par la constitution ${ }^{13}$ qui dispose que les collectivités publiques assurent l'éducation en créant des conditions favorables à cette éducation. Cette disposition constitutionnelle est renforcée par la loi n²003-208 du 07 juillet 2003 portant transfert et répartition de compétences de l'Etat aux collectivités territoriales qui fixent les compétences suivantes transférées en matière d'enseignement et de formation professionnelle à la ville :

- L'élaboration, la mise en œuvre et le suivi des plans urbains de développement des enseignements et de la formation professionnelle en harmonie avec les programmes nationaux

- La construction et la gestion des écoles primaires, maternelles, des crèches et jardins d'enfants, des institutions d'éducation féminine et des centres d'apprentissage en harmonie avec la carte scolaire

- L'Alphabétisation avec le plan d'action national

Le constat avec ces compétences transférées, c'est l'absence du principe de libre administration car les collectivités territoriales ont une compétence liée en la matière. Leurs actions doivent être nécessairement arrimées aux politiques nationales en matière d'éducation au risque de ne pas être acceptées par la tutelle.

À côté de ces compétences, une autre forme de décentralisation existe à travers l'existence des comités de gestion des établissements scolaires $(\text { COGES })^{14}$. Ces comités crées a la faveur de la loi de septembre 1995 relative

\footnotetext{
${ }^{12}$ Article 44 de la loi sur l'orientation de l'organisation générale de l'administration territoriale de 2014.

${ }^{13}$ Article 10, alinéa 2 de la constitution de novembre 2016.

${ }^{14}$ Les COGES sont régis par deux textes le Décret $n^{\circ}$ 2012-488 du 7 juin 2012 portant attributions, organisation et fonctionnement des comités de gestion des établissements scolaires publics, en abrege coges) du 7 juin 2012 et L'Arrêté ministériel Nº80/MEN/CAB du 8 août 2002
} 
à l'Enseignement ${ }^{15}$, sont chargés de veiller à la bonne marche de l'établissement en lui confiant le fonctionnement et la gestion ${ }^{16}$ des activités socio-éducatives, pédagogiques, financières et de l'entretien de l'école. Cette structure regroupe l'ensemble de la communauté éducative ainsi que des représentants des intérêts publics, des activités économiques, culturelles et sociales qui constituent l'environnement de l'établissement. En d'autres termes, à travers les COGES, l'Etat vise l'implication de la communauté et des populations bénéficiaires dans la gestion des écoles. Si les élus des collectivités territoriales en assurent la présidence depuis 2012, ces comités font l'objet dans les faits d'un contrôle hiérarchique de la part du ministre de l'éducation nationale ${ }^{17}$. La présence des collectivités territoriales au sein de ces comités n'a qu'une valeur symbolique.

\section{La rationalité limitée du processus décisionnel dans les politiques éducatives}

Le processus décisionnel devrait être essentiellement structuré par la prise en compte des besoins et des nécessités des administrés. Cependant, les contraintes liées aux impératifs des politiques nationales et les motivations et les intérêts positionnels et matériels du maire qui est l'acteur central du processus se présentent comme des facteurs lourds constituant un obstacle à la rationalité sociale des décisions (Balme \& Brouard, 2005). À côté de ces deux facteurs, l'influence des groupes d'intéret contribue également à éloigner les administrés de la prise de décision.

\section{Le poids des motivations électoralistes dans la mise sur agenda}

L'idée que l'État ou les collectivités apporteraient des solutions à des problèmes économiques et sociaux en agissant au nom de l'intérêt général par la rationalité de son action est une fausse évidence que l'analyse des politiques publiques tente de déconstruire. En effet, de nombreux travaux ont permis par leurs conclusions, de créer la rupture en mettant en cause le naturalisme des faits sociaux et la prétendue rationalité des enjeux politiques. A la vérité, les

${ }^{15}$ LOI n ${ }^{\circ} 95-696$ du 7 septembre 1995 relative à l'Enseignement. Article 8 :

${ }^{16}$ Le COGES a pour mission de contribuer à la promotion de l'établissement où il siège et d'y créer les conditions d'un meilleur fonctionnement en y favorisant son intégration dans le milieu par un appui aux activités socioéducatives et pédagogiques. À cette fin, le COGES est chargé : créer les meilleures conditions de fonctionnement de l'établissement. À ce titre, il doit aider à entretenir couramment les bâtiments et les équipements et aide à sauvegarder le patrimoine et l'environnement. Au niveau des élèves, il doit contribuer et œuvre pour a la promotion de l'hygiène et la santé à l'école avec l'implantation et à la promotion des cantines scolaires. Au plan financier, le COGES recouvre et gère toutes les ressources financières de l'établissement autres que le budget de l'Etat.

17 À travers la direction de l'animation, de la promotion et du suivi des COGES (DAPSCOGES), représentant du ministère de l'éducation national auprès des COGES. 
politiques publiques ne sont pas le résultat d'un processus linéaire et empreint d'objectivité dans la mesure où, en amont de celles-ci, se trouve toute une problématisation qui conditionne les prises de décisions. Ce qui fait dire à $\mathrm{C}$ .W Anderson «qu'élaborer une politique ne signifie pas uniquement résoudre des problèmes, cela signifie également construire des problèmes » (Anderson C. W., 1988). C'est-à-dire que la décision est le résultat d'une confrontation et une articulation de valeurs et des représentations portées par des acteurs qui poursuivent des finalités multiples, non nécessairement réductibles à la résolution du problème.

Ainsi, il ne suffit pas simplement qu'un problème existe pour que l'action publique se mette en œuvre, car l'essentiel se situe dans l'interprétation qui en est faite. En somme, avant de le réguler, il faut que le domaine de l'action publique concerné soit construit et que les enjeux soient sélectionnés et configurés. En somme, l'interprétation revêt une importance capitale parce que les autorités ne peuvent pas prendre en compte tous les problèmes publics pour la simple raison que : "L'attention publique est une ressource rare, dont l'allocation est réglée par la compétition dans un système d'arènes publiques » (Hassenteufel, 2011, p. 51).

Les autorités s'adonnent donc à une sorte de catégorisation, de sélection des dimensions pertinentes des faits sociaux. Cette phase de sélection, au cours de laquelle les pouvoirs publics décident de porter leur attention sur un problème particulier au détriment d'un autre, correspond à la phase de formulation et de mise sur agenda public (Anderson, 1975). On définit l'agenda comme étant : «l'ensemble des problèmes faisant l'objet d'un traitement, sous une forme ou une autre, de la part des autorités publiques » (Philippe Garraud, s. d., p. 27).

En tout état de cause, aborder «l'action publique à partir d'une perspective d'agenda permet de mettre en lumière des logiques de pouvoir, les obstacles culturels, et les freins institutionnels qui empêchent certains sujets d'être pris en compte par les autorités publique » (Kübler \& Maillard, 2009). Dans les collectivités territoriales en Côte d'Ivoire, la faiblesse de l'autonomie institutionnelle que nous avons relevé dans la loi n²003-208 du 07 juillet 2003 portant transfert et répartition de compétences de l'Etat aux collectivités territoriales en matière d'enseignement et de formation professionnelle à la ville constitue le premier obstacle dans la mise sur agenda. En effet, qu'il s'agisse des plans urbains de développement des enseignements, de la construction et de la gestion des écoles et de l'alphabétisation, leur mise en œuvre et leur réalisation sont soumises au respect de l'architecture de la politique nationale. Pourtant, la décentralisation, doit permettre aux collectivités locales de disposer d'une certaine liberté de décision pour définir les normes de leurs actions et les modalités de leurs interventions. D'ailleurs, la gestion des écoles qu'évoque la loi n'est effective ni au plan organique, ni 
au plan matériel. En effet, depuis 1995, les écoles sont gérées par les COGES sous l'autorité du ministère de l'éducation nationale et les élus n'assurent que la présidence des assemblées générales avec des pouvoirs limités ${ }^{18}$.

Deux situations limitent l'influence des communes sur les COGES. Primo , sur plus de 8000 COGES (Ministère de l'éducation nationale de la République de Côte d'Ivoire, 2016) au niveau primaire, environ 3000 reçoivent des subventions du ministère de l'éducation nationale qui exercent un contrôle sur leurs fonctionnement. Au niveau secondaire, ils ne sont pas subventionnés mais reçoivent une quote-part des frais d'inscription en ligne des élèves ${ }^{19}$. Secundo, les ménages sont, de loin, les principaux financeurs des COGES, avec $92 \%$ des activités au niveau secondaire et $73 \%$ au niveau primaire (Ministère de l'éducation nationale de la République de Côte d'Ivoire, 2016). En somme, il n'y a que dans certains cas que des conseils régionaux et mairies subventionnent également les COGES. Les subventions n'étant pas des obligations légales parce que laissées à la discrétion des organes dirigeants des collectivités, le choix des COGES à financer n'est pas toujours un choix naturel et objectif. Si dans l'ensemble, la marie à de faibles chances d'orienter la mise sur agenda des activités des COGES qui sont assujetties aux décisions du ministère et de l'assemblée générale, ce n'est pas toujours le cas en ce qui concerne, ceux qui bénéficient de ses subventions ${ }^{20}$.

Si la mise sur agenda est dans un premier temps structuré par le pouvoir central, dans un deuxième temps, elle est également sous l'emprise des intérêts électoralistes et matériels des élus locaux. Autrement dit, même si une politique éducative locale répond aux exigences de la politique nationale, elle ne fera pas systématiquement l'objet d'une attention particulière de la part du conseil municipal.

À Cocody, l'acteur qui détermine de façon systématique le contenu de l'agenda public et les orientations de l'action publique est le maire. Ainsi, dans la phase de formulation et de mise sur agenda public des politiques éducatives, les logiques de pouvoir qui structurent les motivations de celui-ci constituent des freins a une prise en compte transparente des problèmes publics. Les politiques éducatives constituent en réalité des ressources importantes de la compétition politique (Douillet \& Robert, 2007) que les autorités politiques vont contrôler à travers une main mise sur le processus de décision. En d'autres termes, les politiques éducatives qu'il propose ne sont pas

\footnotetext{
${ }^{18}$ Selon l'article 10 du décret sur les COGES : Le président dirige les débats de l'assemblée générale et préside l'assemblée élective des directeurs d'école d'un groupe scolaire pour la désignation du vice-président de l'assemblée générale.

${ }^{19}$ Celle-ci leur est retournée sans passer par le budget. Ces subventions sont complétées par des contributions des parents et dans certains cas d'ONG, entreprises privées ou individus.

${ }^{20}$ Nous n'avons pas obtenir la liste des COGES qui sont bénéficiaires des subventions de la mairie pour des raisons qui ne nous ont pas été communiquées.
} 
nécessairement le résultat de la volonté des administrés et de l'intérêt général. À travers le contrôle a priori qui est instauré, le processus est loin de promouvoir la démocratie locale et la gestion participative qui sont des modalités importantes de la décentralisation.

Comme nous l'avons souligné précédemment, la préparation d'un projet et la construction d'une politique éducative, est l'affaire exclusive du maire. Si formellement le maire doit recueillir l'avis motivé de la commission des affaires économiques, financières et domaniales et celle des affaires sociales et culturelles, il est quasiment impossible dans les faits que ces commissions donnent un avis défavorable à un projet initié par le maire. Ces commissions fonctionnent exactement comme des caisses d'enregistrement. Une fois la phase de l'examen en commission achevée, le dossier est acheminé sur la table du conseil municipal pour une délibération, un vote sur la base des informations et avis des commissions ${ }^{21}$. Il s'agit en réalité, d'une formalité car il n'y a véritablement pas de débat. La volonté du maire ainsi traduite en projet adopté par le conseil municipal est transmise au préfet d'Abidjan ${ }^{22}$.

La loi $^{23}$ et les principes de bonne gouvernance enjoignent le maire et son conseil municipal à organiser un vote public du budget afférent au projet de telle sorte que tout habitant de la localité puisse assister à la discussion et au vote du budget. Ce citoyen peut même prendre connaissance des documents budgétaires au siège de la collectivité ${ }^{24}$. Or, dans le cadre de notre étude, il nous a été impossible de consulter lesdits documents et selon les informations que nous avons recueillies sur place, le contribuable n'est pas invité à suivre les débats et le vote du budget qui s'en suit. L'une des manifestations de la rationalité limitée du processus décisionnel est le non-respect du contenu de la carte scolaire qui sert à planifier les besoins futurs d'éducation, au niveau local et les moyens à mettre en œuvre pour satisfaire ces besoins ${ }^{25}$. L'exemple du village de Djorogobité ${ }^{26}$, est symptomatique de ce dysfonctionnement ${ }^{27}$.

${ }^{21}$ Généralement, cette phase, la préparation du projet par le Maire, son examen par les Commissions et le vote du Conseil municipal a lieu entre le 16 Juillet et le 30 Septembre de l'année civile.

${ }^{22}$ Le Préfet reçoit en fait les procès-verbaux des séances du Conseil et sa délibération en 12 exemplaires, dans un délai de 15 jours francs à compter de la date de la réunion.

${ }^{23}$ La loi ${ }^{\circ} 2012-1128$ du 13 Décembre 2012 portant organisation des collectivités territoriales dispose en son art.32: «Les réunions des Conseils sont publiques », sauf dans des cas précisés par la loi.

${ }^{24}$ Article 35 de la loi N²012-1128 du 13 Décembre 2012 déjà cité.

${ }^{25} \mathrm{La}$ loi portant transfert de compétences aux collectivités locales fait obligation aux communes de se doter de cet instrument majeur pour aiguiller la construction et la réhabilitation et l'équipement des établissements scolaires.

${ }^{26}$ Ce village est situé non loin du nouveau CHU d'Angré.

${ }^{27}$ Inscrite comme zone prioritaire devant bénéficier d'une école dans la carte scolaire, ce village n'a pu bénéficier de celle-ci qu'en 2018 grâce à la multinationale Orange dans le cadre de la Responsabilité Sociale d'Entreprise (RSE). 
Ainsi, ce sont moins les propriétés intrinsèques d'un problème par exemple sa gravité ou son urgence, ou la mobilisation autour de lui, les ressources de ceux qui le portent et le relaient, les réponses disponibles en termes d'action publique et son adéquation avec des valeurs dominantes dans une société donnée et à un moment donné qui expliquent sa mise sur agenda par des autorités publiques (Hassenteufel, 2010). À côté des dysfonctionnements que nous venons d'énumérer, le processus décisionnel est également affecté par le poids des acteurs non étatiques.

\section{L'influence des acteurs non étatiques}

$\mathrm{Au}$ plan local, les motivations électoralistes des élus entrainent une cohabitation de la vision stato-centré et celle de la vision pluraliste qui prend en compte le rôle et l'influence des acteurs extérieurs (Chabanet \& Guigni, s. d.). Ces acteurs qui constituent des groupes d'intérêts ${ }^{28}$ peuvent être constitués des groupes sociaux et économiques (entreprises) constitués d'acteurs intervenants dans la mise en œuvre concrète de la politique publique ou des groupes de pressions qui ont la capacité de participer à la construction d'un problème public et susciter sa mise sur agenda. Ces groupes sociaux sont déterminants parce qu'ils peuvent donner des consignes de vote en faveur d'un candidat (Dupuy \& Halpern, s. d.). La dimension électorale joue pour deux raisons: D'une part, parce que le groupe représenté forme une clientèle électorale constituée de ressources militantes et politiques numériquement importantes et d'autre part, parce qu'il est présumé avoir la capacité d'influencer un grand nombre d'électeurs neutres. Ces groupes sociaux peuvent également prendre la forme de soutien financier d'un groupe d'intérêt à un parti politique. Ce type de soutien se manifeste au niveau du financement des campagnes électorales. À Cocody, dans le cadre de l'action publique éducative, les groupes d'intérêt prennent la forme d'associations de résidents d'un quartier, de syndics de copropriété, et de comites.

Ces regroupements, formés sur une base volontaire, ont pour but la défense d'une cause, d'un intérêt. Officiellement, il s'agit ici de rapprocher l'école des apprenants en vue de faciliter leur accès et leur maintien dans l'institution mais aussi de permettre aux parents d'encadrer plus aisément leurs

\footnotetext{
${ }^{28}$ On désigne généralement par groupes d'intérêt un ensemble d'acteurs plus ou moins organisés qui cherche à influencer les pouvoirs publics dans un sens favorable aux préoccupations sociales qu'elle prend en charge, sans chercher à en faire partie (ce qui serait le propre de l'administration) ni à exercer ou participer directement à l'exercice du pouvoir politique, contrairement aux partis. Les groupes d'intérêt se distinguent des partis politiques en ce qu'ils, défendent d'une part, des intérêts plus spécifiques (voire sectoriels) et d'autre part, ils ne participent pas directement à la compétition électorale, mais interviennent de façon variée dans la vie politique à travers des protestations, de la production d'expertise, etc.
} 
enfants. À cet effet, ces groupes d'intérêt entrent en négociation avec la municipalité en vue de faire inscrire cette volonté sur l'agenda.

Les premiers groupements sont les COGES que nous avons évoqués dans les développements précédents. Pour beaucoup d'observateurs, ces comités sont des caisses noires qui élèvent des cotisations annexes exorbitantes (Zadi, 2020). En effet, aujourd'hui il est indéniable que l'image : « Des COGES semble être ternie par plusieurs maux, ceux-ci ont pour noms : conflit de compétence entre les différents acteurs, méconnaissance des textes, cotisations aussi bien inopportunes qu'abusives, détournements des fonds collectés, mauvaise gestion caractérisée. Par ailleurs, on assiste à la présence des mêmes personnes dans plusieurs comités de gestion de la localité. (...). Ces dérives entrainent le désintérêt total des parents, des enseignants et des élèves qui se manifeste par l'absence aux réunions, aux activités organisées par les responsables des COGES, la difficulté à justifier les cotisations exigées pour le bon fonctionnement des COGES » (Kei, 2010, p. 42-43). Cependant, face aux dérives constatées, les élus locaux font preuve de passivité dans beaucoup de cas dans la mesure où les assemblées générales sont composées d'importantes entités communautaires dont le suffrage peut faire la différence. Le maire qui préside l'assemblée générale qui est l'organe de décision est celui qui dirige les débats qui autorisent les cotisations exceptionnelles qui créent les polémiques. En d'autres termes, le maire est témoin des charges financières supplémentaires imposées à ses administrés et qu'il peut soulager en subventionnant les comités sur le budget de sa commune. A défaut de cette solution, les élus se rangent derrière les décisions et les manœuvres des COGES. Dans les faits et selon les données de l'enquête, après s'être assuré de la fidélité des représentants des acteurs envers sa personne, le maire accède à leur demande ou ferme les yeux sur leurs agissements. Cette faible implication des élus dans la gestion de l'école avait suscité un séminaire ${ }^{29}$ en octobre 2013, en vue de réclamer leur implication effective dans la gestion et le fonctionnement des comités de gestion des établissements scolaires (Coges). À cette occasion, le représentant du ministre de l'intérieur : «a instruit les élus à inscrire l'appui des Coges dans leur budget et en faire une priorité » (Jean, 2013). Récemment d'ailleurs, la maire de la commune de Tiassale a tout simplement supprimé les cotisations des parents au profit des COGES et en compensant ce manque à gagner par une subvention (Éducation/Tiassalé : Le maire met fin aux cotisations Coges | FratMat, s. d.). Pour l'élu, la commune par cette décision venait soulager les parents d'élèves parce que les frais COGES: « en plus de peser sur le pouvoir d'achat des parents d'élèves, ont montré leur limite au regard de l'état de dégradation de

\footnotetext{
${ }^{29}$ Ce séminaire organisé par le ministère de l'Éducation nationale, à travers le Service national d'animation de promotion et de suivi des Coges (Snaps-Coges).
} 
la plupart des écoles de la commune et surtout de l'absence de transparence dans leur gestion ».

En ce qui concerne le poids des syndics et les associations, le cas de l'association des habitants de la Cité Joël Dervain (appelé aussi Cité SIR) à Akouédo 6 palmeraie nord illustre parfaitement cette situation. Cette cité inaugurée en 2009 au profit du personnel de la Société Ivoirienne de Raffinage (SIR) a fait la demande d'un groupe scolaire conformément aux exigences de la carte scolaire sans suite jusqu'en 2013. Avec l'inflation de la population de la zone ${ }^{30}$ à la faveur de la crise post-électorale, les habitants de la cité et ceux des alentours se sont constitués en association pour relancer l'idée de l'école. En 2013, des démarches ont été entreprises auprès des nouvelles autorités municipales qui ont réagi favorablement et cela, en toute urgence ${ }^{31}$, alors même que le Programme Triennal 2013-2015 avait été déjà approuvé.

\section{À côté des groupes de pression, les entreprises constituent également des acteurs importants}

Ces unités économiques produisent pour la vente ou distribuent des biens et services moyennant paiement et cela, conformément à des objectifs définis par leur direction. Mais ce n'est qu'à partir du moment où l'État ou une collectivité territoriale fait appel aux entreprises que celles-ci deviennent des acteurs des politiques publiques. Le processus par lequel l'État ou une collectivité territoriale fait appel aux acteurs privés s'appelle, de façon générique, la contractualisation des politiques publiques. La forme la plus usitée est l'appel d'offre. L'appel d'offre est un mode de passation des marchés publics, dans lequel la personne publique choisit l'offre économiquement la plus avantageuse, sur la base de critères objectifs préalablement portés à la connaissance des candidats, et sans négociation avec ceux-ci. Or à Cocody, le choix des entreprises se fait dans des conditions opaques. Aucun agent de la Mairie n'a pu nous décrire de façon précise le mode de passation des marchés avec les entreprises. Tous se sont contentés de nous affirmer que cela relève du pouvoir discrétionnaire du Maire. Ainsi sur la période 2013-2017, ce sont des marchés d'un coût total de sept cent soixante-treize millions huit cent cinquante-trois mille huit cent soixante-onze francs CFA (773.853.871frs CFA) qui ont été concédés à des entreprises exerçant pour la plupart dans le domaine du bâtiment avec des procédures irrégulières. En effet, le rapport 2017 de l'autorité de régulation des marchés publics (ANP) a relevé 7 marchés irréguliers sur 11 audités, soit $64 \%$ des marchés. Cette absence totale de transparence sur la question pourrait s'expliquer que par la recherche d'intérêts matériels et positionnels.

\footnotetext{
${ }^{30}$ Les alentours de la cité ont aussi enregistré une arrivée massive de population.

${ }^{31}$ La construction de cette école est brandie par le Maire comme son action personnelle au profit des populations sur le site "jevotengoan.com".
} 


\section{Les contraintes liées à la mise œuvre des politiques éducatives}

$\mathrm{Au}$ niveau de la mise en œuvre, un ensemble de facteurs exerce également une influence sur les politiques publiques. Il s'agit des institutions qui peuvent être définies comme : «Les règles formelles et informelles, les normes, les précédents ainsi que les facteurs organisationnels qui structurent le comportement politique » (Pomey et al., 2010, p. 709). Les institutions se rapportent aux structures gouvernementales c'est-à-dire à la nature du régime et au type d'organisation politique d'un pays. Ainsi, les structures gouvernementales peuvent façonner et entraver de maintes façons le développement et les choix de politiques. Dans le cas de cette étude, le contrôle politique de la mise en œuvre des politiques éducatives par la tutelle restreint les marges de manœuvre de l'organe de décision municipal. Au final, la mise en œuvre de ces politiques est subordonnée aux logiques du pouvoir central et de la majorité présidentielle. Ces logiques se manifestent par une insuffisance des budgets et une faiblesse structurelle.

\section{La faible autonomie financière}

L'autonomie financière apparaît comme un corollaire du principe de la libre administration des collectivités locales. En effet, la concrétisation de ce principe de libre administration suppose que les collectivités locales soient dotées de compétences juridiques pour décider de la nature, de la structure et du niveau de leurs ressources et de leurs charges financières, et qu'elles en aient la maîtrise. La faible autonomie financière se manifeste à travers deux situations : la dépendance administrative vis à vis de la comptabilité publique générale et la faiblesse du budget de la commune.

\section{La dépendance financière de l'administration centrale}

La loi du 26 décembre 2003 portant régime financier, fiscal et domanial en Côte d'ivoire ne consacre pas expressément l'autonomie financière des collectivités locales. Néanmoins, ce principe y est sous-jacent. Or à l'instar de l'Etat, les collectivités locales peuvent recourir à l'impôt pour financer leurs dépenses. Et la loi du 26 décembre 2003 portant régime financier, fiscal et domanial des collectivités territoriales en Côte-d'Ivoire citent parmi les ressources des collectivités locales, les recettes fiscales. Toutefois, les collectivités locales en Côte d'Ivoire ne disposent pas d'une autonomie fiscale car, elles n'ont pas le pouvoir de créer un impôt ${ }^{32}$, d'en déterminer l'assiette, le taux et les modalités de recouvrement. Les ressources

\footnotetext{
${ }^{32}$ En vertu du principe de légalité fiscale, expression juridique du principe du consentement de l'impôt découlant de l'article 14 de la Déclaration des droits de l'Homme et du citoyen, il revient au législateur de fixer le régime de l'impôt local.
} 
fiscales ${ }^{33}$ des collectivités locales proviennent donc essentiellement du partage des impôts étatiques, du produit des impôts locaux dont le régime est fixé par le législateur ou encore d'impôts locaux dont elles peuvent déterminer le taux dans les limites déterminées par la loi. Or, il ne peut y avoir : "une libre administration des collectivités locales sans une véritable autonomie fiscale locale, c'est-à-dire sans que les autorités locales puissent décider du niveau de la pression fiscale locale » (Ovono, 2012, p. 6). En effet, l'autonomie fiscale locale présente plusieurs avantages : «D'abord, elle donne un fondement à la démocratie locale en créant un lien entre les citoyens et les collectivités locales. Ensuite, sur le plan économique, le pouvoir fiscal conféré aux collectivités locales leur permet en principe de lier le montant des impôts qu'elles prélèvent à celui des services publics qu'elles offrent, sous le contrôle de l'électeur contribuable» (Ovono, 2012, p. 6) . Au niveau des investissements, des dépenses et les charges le pouvoir de décision de la mairie connaît aussi d'importantes restrictions tenant soit à l'existence de dépenses réglementées ou interdites, soit à l'imposition des dépenses obligatoires. Les opérations de dépenses doivent respecter les principes et règles du droit budgétaire et de la comptabilité publique et celles du code des marchés publics.

À côté de cette restriction, il y a la question liée au contrôle administratif exercé sur les décisions budgétaires des collectivités territoriales. Ce contrôle qui est une exigence constitutionnelle ne devrait pas en principe affecter l'autonomie des collectivités. Cependant, l'instauration d'un contrôle a priori en Côte d'Ivoire n'est pas de nature à garantir le principe de libre administration car il prend souvent la forme d'une autorisation préalable de l'autorité de tutelle. En Côte d'ivoire, aux termes des articles 18 et 19 de la loi du 26 décembre 2003 portant régime financier, fiscal et domanial des collectivités territoriales, le budget voté par le conseil est transmis par le maire, à l'autorité de tutelle au plus tard dans les quinze jours francs à compter de la date de vote ${ }^{34}$.

Ainsi, lorsque le maire fait endosser son projet par le conseil municipal, le dossier est envoyé au préfet. Lorsqu'il accuse réception du

\footnotetext{
${ }^{33}$ Dans le premier cas, le commun est bénéficiaire du partage du produit d'un impôt national en recevant les quotes-parts d'impôts nationaux sont versées aux collectivités locales. Dans le second cas, les collectivités locales perçoivent l'intégralité du produit d'un impôt prélevé sur une matière imposable locale, mais sans disposer du pouvoir d'en déterminer le taux.

${ }^{34} \mathrm{~L}$ 'article 19 donne la possibilité à l'autorité de tutelle d'effectuer d'office et sans renvoi du budget, les corrections de forme.
} 
dossier, le préfet ${ }^{35} \mathrm{~d}$ 'Abidjan à son tour l'examine ${ }^{36}$ puis adresse une copie du dossier au ministre de l'intérieur par le truchement du directeur général de la décentralisation et du développement local.

Le préfet saisit également le ministre chargé de l'économie et des finances via la commission des programmes et budgets pour avis. En définitive, le dossier atterrit sur la table du ministre en charge de l'administration du territoire qui décide en dernier ressort. Cette décision du ministre intervient sous la forme d'un arrêté ${ }^{37}$. On pourrait reconstituer schématiquement le processus de la prise de décision selon les données du tableau suivant.

Tableau 1 : Tableau décrivant les étapes et les acteurs étatiques intervenant dans la décision

\begin{tabular}{|l|l|}
\hline Étapes & Acteurs étatiques impliqués dans le contrôle de tutelle a priori \\
\hline $\mathbf{1}$ & Préparation du projet par le Maire \\
\hline $\mathbf{2}$ & Examen par les Commissions \\
\hline $\mathbf{3}$ & Délibération du Conseil municipal et fixation de la priorité de l'opération \\
\hline $\mathbf{4}$ & Transmission au Préfet \\
\hline $\mathbf{5}$ & Visa du Préfet \\
\hline $\mathbf{6}$ & Transmission au directeur de la décentralisation et du développement local \\
\hline $\mathbf{7}$ & Avis de la Commission des programmes et des budgets \\
\hline $\mathbf{8}$ & Transmission au Ministre chargé de l'économie \\
\hline $\mathbf{9}$ & Transmission au Ministre du Budget \\
\hline $\mathbf{1 0}$ & Visa du Ministre chargé de l'Économie \\
\hline $\mathbf{1 1}$ & Visa du Ministre chargé du Budget \\
\hline $\mathbf{1 2}$ & $\begin{array}{l}\text { Décision finale du Ministre d'État en charge de l'administration du territoire } \\
\text { sous la forme d'un arrêté }\end{array}$ \\
\hline
\end{tabular}

Finalement, l'on constate qu'au plan financier et institutionnel, le processus est pris en otage par le pouvoir central si bien que les choix effectués relèvent plus d'un calcul rationnel, celui de renforcer la légitimité de la majorité au pouvoir.

C'est pourquoi en dépit des difficultés financières, l'on constate dans certaines situations que les dépenses publiques de transfert sont plus élevées

${ }^{35}$ Au terme cette étape, le Préfet peut adopter deux (02) attitudes : soit il renvoie le projet au Maire, soit il l'approuve si celui-ci est conforme aux exigences. Quand le projet est conforme aux exigences, il est transmis au Ministre auprès du 1er Ministre chargé de l'Économie et des Finances puis au Ministre auprès du 1er Ministre chargé du Budget qui tous deux apposent leur visa.

${ }^{36}$ Il examine seulement la conformité des documents aux exigences légales.

${ }^{37}$ Par exemple les projets éducatifs initiés sous l'empire du Programme Triennal 2015-2017, ont été autorisés par le Ministre de l'intérieur à travers l'arrêté $\mathrm{n}^{\circ} 113 / \mathrm{memis} / \mathrm{dgddl} / \mathrm{dtef} / \mathrm{sdbf}$ du 15 Avril 2015 portant approbation et règlement du Programme Triennal 2015-2017 de la Commune de Cocody signé du Ministre de l'Intérieur de l'époque. 
que les années d'élections. Mieux, pour aller dans le sens de Maillard, « la proximité d'une échéance peut conduire des gouvernements à hâter la mise en œuvre d'un dispositif enfin d'en retirer des bénéfices politiques » (Kübler \& Maillard, 2009, p. 48-49). Une telle situation s'observe avec les investissements effectués avec les programmes triennaux de la mairie dans la construction des établissements scolaires. Les deux (02) programmes triennaux 2013- 2014- $2015^{38}$ et le Programme Triennal 2015- 2016$2017^{39}$ ont enregistré la construction et la réhabilitation d'école qui ont servi d'arguments de campagne au maire sortant. C'est d'ailleurs cette situation que la ministre de l'éducation nationale a éloquemment caricaturé à l'occasion d'un meeting qu'elle animait le mardi 9 octobre 2018 à Tiébissou en faveur des candidats de son groupement politique le RHDP. S'adressant à la foule, elle leur a recommandé de demander ceci aux candidats de l'opposition qui demandaient leurs suffrages :

"Tout ce que tu veux faire là c'est bien mais est ce que toi tu connais le premier ministre Gon Coulibaly? Tu lui demandes, oui mon frère, tout ce que tu veux faire là c'est bien mais estce que toi là tu peux rencontrer le président de la République Alassane Ouattara? Faut lui demander mais toi c'est le président qui t'envoie ici pour venir travailler pour nous ou c'est le premier ministre? S'il ne répond pas à la question, ne l'écoutez plus parce que c'est le maire qui vient mettre en cuvre la politique sociale du gouvernement, c'est le maire qui vient mettre en place le programme de développement du gouvernement, le maire là, même le budget du maire c'est le gouvernement qui lui donne, tous les projets que le maire réalise c'est le premier ministre et le gouvernement qui lui permettent de faire ça, mais si toi le gouvernement t'as pas choisi, le premier ministre t'a pas choisi et le président te connait pas, tu peux faire quoi pour les gens? Rien ». 1.

\footnotetext{
${ }^{38}$ Le Programme Triennal 2013-2015, comporte douze (12) actions estimées à trois cents trente et un million de francs CFA (331.000.000 Frs CFA) ${ }^{38}$ et vingt-six (26) opérations évaluées à un milliard sept cents cinquante-cinq millions de francs CFA (1.755.000.000 Frs CFA) pour la période indiquée. Ce programme est exclusivement financé sur fonds propre de la commune.

${ }^{39}$ Le Programme Triennal 2015-2017, comporte dix-huit (18) actions estimées à un milliard six-cents vingt millions de francs $\mathrm{Cfa}(1.620 .000 .000 \mathrm{frs} \mathrm{Cfa})$ dont cent quatre-vingts quinze millions de francs Cfa (195.000.000 frs Cfa) affectés aux prises en charge scolaire et universitaire et quarante-six (46) opérations d'un coût total de trois milliards deux-cents trente-huit millions cinq cents mille francs Cfa (3.238.500.000 frs Cfa).
} 
La faible autonomie institutionnelle et financière débouche sur des difficultés financières des administrations en charge des politiques éducatives.

\section{La faiblesse des budgets de la mairie}

L'administration centrale constitue la source principale de financement ${ }^{40}$ de l'éducation en Côte-d'Ivoire quand on considère l'ensemble des administrations publiques. Le financement par le programme présidentiel d'urgence (PPU) ${ }^{41}$ qui intervient à partir de 2012 se situe à un niveau important des dépenses de l'administration centrale. En ce qui concerne les collectivités territoriales (Conseils généraux et Mairie), leur apport est dérisoire. Sur, la periode (2006-2014) (Ministère de l'éducation nationale de la République de Côte d'Ivoire, 2016), par exemple, elles n'ont exécuté au plus que $1 \%$ du total des dépenses d'éducation des administrations publiques sur la période. En dépit de cette prépondérance de l'administration centrale dans le financement de l'éducation, les collectivités territoriales telle que la mairie de Cocody éprouvent dans leur ensemble des problèmes de trésorerie (N'tchuvi \& Aloko, 2018). Afin de les appuyer dans la prise en charge de leur fonctionnement et dans la réalisation de leurs investissements, l'Etat alloue annuellement aux collectivités, des dotations ${ }^{42}$ inscrites au budget de l'Etat comme le prévoit la loi $\mathrm{n}^{\circ} 2003-489$ du 26 décembre 2003 portant régime financier, fiscal et domanial des collectivités territoriales. Des subventions ${ }^{43}$ sont allouées à l'ensemble des collectivités par l'Etat, à l'exception du District Autonome d'Abidjan et de ses dix(10) principales communes qui ne peuvent en bénéficier qu'exceptionnellement et pour des opérations spécifiques, compte tenu de leur grande capacité de mobilisation de ressources propres et de l'importance de la quote-part d'impôts partagés qui leur est reversée. Elles constituent une contribution du budget de l'Etat à certaines collectivités qui en bénéficient en plus des ressources propres collectées localement et leur quotepart des impôts partagés. La mairie de Cocody n'en bénéficie

\footnotetext{
${ }^{40}$ Les financeurs, qui sont les unités qui financent le système éducatif, divisées en trois grands groupes : les administrations publiques (centrale, régionales et locales), le secteur privé (ménages, sociétés et institutions à but non-lucratif comme les ONG locales) et le Reste du monde (prêts et dons des bailleurs et partenaires techniques, ONG internationales). Les producteurs, qui sont les unités qui produisent les services éducatifs, divisées en trois grands groupes : les établissements d'enseignement publics, les établissements d'enseignement privés, et les entités administratives.

${ }^{41}$ Le Programme Présidentiel d'Urgence est une cause importante de l'augmentation des dépenses en éducation. Il s'évalue entre 36900 et 46500 millions de FCFA depuis 2012.

${ }^{42}$ Ces subventions sont octroyées sur la base d'un ensemble de critères dont la population et la spécificité de la commune.

${ }^{43}$ Ainsi, pour la gestion 2017, ce sont cent quatre-vingt-sept (187) Communes, trente-et-une (31) régions, le District Autonome de Yamoussoukro et, à titre exceptionnel, le District Autonome d'Abidjan qui ont bénéficié de subventions de l'Etat.
} 
qu'exceptionnellement sur la base des éléments que nous avons évoqué dans les développements précédents ${ }^{44}$. Ce dont bénéficie la commune de Cocody a l'instar des autres communes d'Abidjan, c'est sa quote-part des impôts partagés. Cependant, le partage de ces impôts entre l'Etat et les collectivités territoriales, a consisté pour l'Etat, jusqu'à l'exercice budgétaire 2015, à se dessaisir de la quote-part devant revenir aux collectivités sans la comptabiliser dans la loi de finances. Cette situation a changé depuis la loi de finances 2016 car la quote-part d'impôts partagés à reverser aux collectivités territoriales est désormais inscrite dans le budget de l'Etat. Ce changement vient alourdir les procédures administratives des collectivités territoriales pour rentrer en possession de leurs budgets. Cette situation pose la question de la dépendance de la mairie envers l'État avec le mécanisme avec l'unicité des caisses qui freine la réalisation à temps des opérations éducatives dans la mesure où la mairie doit indéfiniment atteindre que l'État lui reverse sa part ${ }^{45}$.

À côté de cette situation préjudiciable, l'autre constat qui se dégage comme pour l'ensemble des communes d'ailleurs, les ressources financières bien qu'importantes comparativement à la majorité des communes de Côted'Ivoire demeurent insuffisantes (Assepo, 2002) à l'échelle de Cocody. La modicité des montants reversés à la commune constitue un obstacle au financement idoine de la politique éducative. Les sources de financement de la commune de Cocody sont diverses. Ils sont constitués entre autres des recettes fiscales, des recettes des prestations de service, des revenus du patrimoine, des aides de l'État, des fonds de concours, des aides extérieures. Les recettes fiscales sont constituées du produit des impôts rétrocédés et des taxes fiscales. Quant aux recettes de prestation de services, ces recettes sont constituées des taxes rémunératoires et des redevances. Il s'agit des recettes des services généraux (légalisation de signatures et certifications, délivrance de livrets de famille, etc.), des recettes des services de la commune (autorisation de construire, taxe d'enlèvement des ordures ménagères, services funéraires), des recettes des services culturels (par exemple location de salles.), des recettes des services économiques (par exemple taxe payée par les commerçants sur les marchés).

Les revenus du patrimoine, pour leur part, sont des droits que perçoit la commune en cas d'occupations temporaires de son domaine public. En ce qui concerne les fonds de concours, ce sont des apports financiers provenant de personnes privées ou publiques en vue du financement de certains

\footnotetext{
${ }^{44}$ Les aides de l'État au budget de la commune qui sont faites sous la forme de dotations. Ce sont des aides que l'État accorde automatiquement à la commune. La plus importante de ces dotations est la dotation générale de fonctionnement (DGF).

${ }^{45}$ C'est par exemple ce que l'on a observé en 2013, le budget de la commune a été approuvé et signé quasiment en fin d'année. En 2014, il a fallu attendre la fin du 2ème trimestre de l'année pour l'approbation, donc la disponibilité des fonds.
} 
investissements publics. Les aides extérieures sont des transferts financiers en nature ou en numéraire en provenance de l'extérieur, d'autres États, organisations internationales, publiques ou d'organismes privés.

Notons cependant que, dans la pratique, ce sont les impôts et les taxes, donc les recettes fiscales qui constituent des sources de financement propres de la commune de Cocody, celles sur lesquelles elle s'appuie pour mener à bien ses différentes politiques dont la politique éducative. Concernant l'impôt foncier, cet impôt annuel constitue une source importante de revenu dans le contexte de Cocody, vu les opérations immobilières qui y ont cours chaque année. Cependant, seulement $30 \%$ sont reversées à la commune de Cocody et $70 \%$ à l'État ${ }^{46}$. La clé de répartition de l'impôt synthétique depuis 1994 reste la même à savoir $40 \%$ pour la commune et $60 \%$ à l'État. Conscient des difficultés liées au principe de l'unité de trésorerie, au rang desquelles l'insuffisance des ressources financières allouées aux communes (Bede, 2017), l'État de Côte-d'Ivoire a prévu des assouplissements au principe. Il s'agit des de placements ${ }^{47}$ que les collectivités locales sont autorisées à faire et du dépôt auprès de certains organismes en contre parti d'un intérêt. Ces aménagements sont loin d'avoir régler le problème

Tableau 2 : Tableau récapitulatif des actions éducatives de la Mairie de Cocody entre 2013-2017

\begin{tabular}{|c|c|c|}
\hline $\begin{array}{l}\text { Programme } \\
\text { triennal }\end{array}$ & Constructions & $\begin{array}{l}\text { Réhabilitations } \\
\text { et équipements }\end{array}$ \\
\hline \multirow{2}{*}{$\begin{array}{l}\text { 2013-2014- } \\
2015\end{array}$} & $\begin{array}{l}\text { Construction d'une école } \\
\text { maternelle au quartier Syninfo. } \\
\text { Coût de l'opération : } \mathbf{5 5 . 0 0 0 . 0 0 0 f r s} \\
\text { CFA }\end{array}$ & $\begin{array}{l}\text { Acquisition de mobiliers } \\
\text { scolaires pour les Groupes } \\
\text { Scolaires } 2 \text { Plateaux et Angré. } \\
\text { Coût de l'opération : } \\
\text { 50.000.000frs CFA }\end{array}$ \\
\hline & $\begin{array}{l}\text { Construction d'une école primaire } \\
\text { à Blockhauss. Coût de l'opération : } \\
\mathbf{5 0 . 0 0 0 . 0 0 0 f r s ~ C F A ~}\end{array}$ & $\begin{array}{l}\text { Construction d'un hangar au } \\
\text { Groupe Scolaire Riviera Golf. } \\
\text { Coût de l'opération : } \mathbf{1 5 . 0 0 0 . 0 0 0} \\
\text { frs CFA }\end{array}$ \\
\hline $\begin{array}{l}\text { 2015-2016- } \\
2017\end{array}$ & $\begin{array}{l}\text { Construction d'un établissement } \\
\text { pour la petite enfance à Génie } 2000 \\
\text { Nord, à Akouédo-extension. } \\
\text { Coût de l'opération : } \\
\mathbf{7 0 . 0 0 0 . 0 0 0 . f r s ~ C F A ~}\end{array}$ & $\begin{array}{l}\text { Réhabilitation des écoles } \\
\text { primaires publiques de la } \\
\text { commune (07 écoles au total). } \\
\text { Coût de l'opération : } \\
\mathbf{2 1 0 . 0 0 0 . 0 0 0 f r s ~ C F A ~}\end{array}$ \\
\hline
\end{tabular}

${ }^{46}$ Ces chiffres ont été communiqués par le Dr Albert François Amichia, maire de la commune de Treichville, lors d'une conférence organisée par la Ligue des Enseignants d'HistoireGéographie de Côte-d'Ivoire sous le thème : «Le rôle de la commune dans la Côte-d'Ivoire émergente à l'horizon 2020 ». Cette conférence s'est tenue le 25 août 2014 à Yamoussoukro, http://news.abidjan.net/h/506290.html, consulté le 12 juillet 2018

${ }^{47}$ Il s'agit d'abord des placements budgétaires portant sur des excédents budgétaires. Il s'agit ensuite des placements de trésoreries portant sur les fonds d'emprunt momentanément utilisés. 
On constate avec le tableau que de 2013 à 2017, le budget est évalué à plus de huit milliards deux-cents millions de francs CFA (8.200.000.000frs CFA) répartis comme suit : pour le fonctionnement à sept milliards vingt-deux mille cent quatorze (7.000.022.114 Frs CFA) et pour l'investissement à un milliard cent soixante-dix-sept millions huit cent quatre-vingt-six mille (1.177.886.000frs CFA). Sur ce budget, sur la période 2013-2015, c'est la somme de sept cent soixante-treize millions huit cent cinquante-trois mille huit cent soixante-onze francs CFA (773.853.871 Frs CFA) soit 13,13\% du budget total d'investissement qui a été investie au titre de la politique éducative. C'est pourquoi à l'occasion des vœux formules à l'endroit de ces administrés le 18 février 2018, on pouvait entendre le maire sortant dire: "Au niveau de l'éducation, sachez que malgré les contraintes budgétaires, nous avons construit de nombreuses écoles et réhabilitées les établissements qui attendaient de l'être. À cela, il faut ajouter plus de 1000 bourses offertes à nos élèves et étudiants, élite de demain ».

\section{Les difficultés structurelles}

Les politiques éducatives locales si elles contribuent au service public de l'enseignement au plan national, restent avant tout une affaire de spécialistes et de structures adéquates. Ainsi, les collectivités locales devraient non seulement se doter de structures spécialises en charge de l'éducation qui doivent impérativement collaborer avec le ministère technique qui est le ministère de l'éducation nationale. Or la sous-direction en charge de l'éducation et de la promotion humaine qui est chargé de mettre en œuvre la politique éducative de la mairie de Cocody est en sous-effectif. Seulement, en dehors de quelques agents administratifs et financiers mis à la disposition de la commune par l'État, l'immense majorité du personnel de la mairie est recrutée directement par le Maire. Les critères qui président au recrutement étant le résultat du clientélisme, il s'ensuit, outre l'incompétence de certains agents, une mauvaise répartition des agents dans les différents services de la Mairie. Ainsi, à la mairie de cocody, pour le compte de la sous-direction éducation et promotion humaine en charge de toutes les questions et projets liés à l'éducation (y compris l'alphabétisation), à la culture, au sport, au tourisme et aux loisirs compte que deux (02) agents sur les trois cents agents (300) qui émargent sur le budget de la commune.

Cette insuffisance numérique du personnel contrarie gravement la mise en œuvre d'une véritable politique éducative. Il faut également ajouter à cette faiblesse la formation inadaptée ${ }^{48}$ des agents de la mairie de Cocody en charge

\footnotetext{
${ }^{48}$ Par exemple, M. Dakoury, Sous-directeur du Service Éducation a une formation initiale de Technicien Supérieur en Gestion Commerciale. En plus de vingt ans de présence 48 à la Mairie de Cocody, il aura travaillé dans quasiment tous les services, avant d'arriver à la tête de la Sous-direction Éducation. Bien qu'il soit pétri d'expérience, de qualités intellectuelles
} 
de gérer les questions éducatives. À la mairie de Cocody, les agents en charge des questions d'éducation ne sont pas formés au pilotage des politiques publiques. L'Éducation, dans sa séquence pilotage des politiques, doit être véritablement l'affaire d'experts et autres professionnels de la question, ayant une vision large des choses tant sur la question de l'Éducation que mobilise des savoirs transversaux allant de la démographie à la psychologie en passant par l'économie et l'analyse des politiques publiques. Dans le cadre du pilotage des politiques éducatives locales, deux sources de connaissance doivent être mobilisées, pour mener à bien les projets et qui font défaut, bien des fois, aux acteurs étatiques locaux: outre la gestion classique des projets et ses déclinaisons ${ }^{49}$, l'analyse des politiques publiques ${ }^{50}$ et l'éthique de l'action publique, c'est-à-dire la connaissance des valeurs et principes qui sous-tendent le service public de l'enseignement. Ainsi, la faiblesse de la collaboration avec les services de l'éducation nationale et le manque de compétences en matière de politiques éducatives peut priver les réalisations de projets éducatifs de l'apport technique nécessaire. Le risque avec une telle configuration, c'est le fait qu'elle puisse aboutir à une hégémonie des motivations politiques, électoralistes et des intérêts matériels dans le fondement des décisions sur la rationalité sociale ${ }^{51}$.

\section{Conclusion}

Le maire actuel de la commune de Cocody, monsieur Yacé Jean Marc à l'occasion des campagnes électorales des élections municipales de 2018 qu'il a remportées établissait le diagnostic du secteur éducatif de la commune en ces termes.
"Il faut une véritable politique de l'Éducation à Cocody. Elle doit être axée sur un renforcement des services publics. En effet, la construction et l'entretien des classes d'établissements publics sont inscrites au budget communal. L'école publique n'est plus ce qu'elle était il y a quelques années. De ces beaux

\footnotetext{
indéniables (c'est un tribun hors norme), de qualités humaines faisant de lui un leader syndical respecté, la question du pilotage des politiques éducatives lui est complètement étrangère. Or de nos jours, avec la complexification des questions liées à l'Éducation, le pilotage des politiques éducatives requiert des compétences techniques et technologiques avérées.

${ }^{49}$ Nous faisons référence ici à la Gestion Axée sur le Résultat (GAR), et le suivi-évaluation des projets, entre autres.

${ }^{50}$ Ces dernières années, les spécialistes des politiques publiques ont développé de nombreux concepts, modèles et approches. Il ne s'agit pas ici d'imposer à l'agent municipal de connaitre toutes les théories et autres concepts de l'Analyse des politiques publiques. Il s'agit plutôt d'en apprendre les notions basiques indispensables à l'exécution de ses missions.

${ }^{51}$ Avec une telle distance, on comprend difficilement comment les décideurs prendront en compte, le contenu de la carte scolaire, document majeur dans la fabrique des politiques éducatives.
} 
locaux aux allées fleuries, aux murs et aux toilettes propres il ne reste plus rien. Les salles de classes sont pléthoriques, le système d'électrification défaillant et l'enseignement délivré au rabais. Il est clair que les établissements publics de Cocody échappent complètement à la modernité et à l'évolution. Pourtant, le fonctionnement et la gestion de l'école publique comme mentionné plus haut, incombent à la commune »

Ce que l'élu dénonce n'est autre chose que la crise de l'action publique éducative dans la commune. C'est-à-dire que, l'évaluation des politiques éducatives locales aboutit à des résultats qui sont loin de ceux initialement visés (Perret, 2014). Il est donc question de vérifier que la mise en œuvre pratique d'un dispositif correspond bien au cahier des charges défini par ses concepteurs, ce qui suppose notamment que les bénéficiaires potentiels de la mesure soient informés et que les agents chargés de l'appliquer soient correctement formés. La bonne articulation de ces différentes phases dépend étroitement du cadre institutionnel dans lequel les politiques publiques sont évaluées. Ce sont tous ces écarts que nous avons tenté de montrer à travers notre étude sur la décentralisation des politiques éducatives à Cocody.

L'étude a abouti à la conclusion que l'évaluation des politiques publiques éducatives peut s'analyser en termes d'inefficacité. L'action publique locale est encore dominée par le modèle «top down» et des approches etatocentré. C'est à dire qu'on conçoit encore l'action publique comme une intervention d'autorités dotées de la puissance publique ou un ordre, un commandement, qui aurait vocation à être transmis et exécuté sans la moindre interférence. Pourtant, une approche centrée sur les sommets de l'État ne peut donner à avoir que les aspects les plus nobles et spectaculaires de l'action. En d'autres termes, les résultats de l'action publique sont présentés avec une mention spéciale sur le rôle des gouvernants qui sont auréolés de superlatifs de leaders héroïques, visionnaires, réformateurs courageux. Cette vision est complétée par une théâtralisation des décisions, des moments historiques qui transforment le cours des politiques publiques et le sort des populations. Alors que : «Cette conception du décideur rationnel et puissant est le plus souvent un leurre, un masque, de processus complexe ou les acteurs sont inscrits dans des trajectoires, des réseaux, des intérêts et des institutions » (Lascoumes \& Galès, 2018, p. 3). En effet, l'étude en abordant la question sous l'angle de du choix rationnel, montre que les politiques publiques éducatives sont le résultat d'individus influencés par des incitations et de sanctions font des choix dans le but de maximiser leur intérêt, leur satisfaction, ou des préférences et non des buts. Alors, la démocratie locale qui inclut une gestion participative (Gerstle, s. d.), étant absente du processus, la rationalité 
sociale et l'intéret général semblent apparaitre comme des finalités dérisoires dans l'action publique éducative face aux logiques propres des élus. Au final, le processus décisionnel, la formulation et la mise sur agenda des problèmes liés à l'éducation sont plus portés vers une rationalité électorale et matérielle que vers une rationalité fondée sur la satisfaction de l'intérêt général $\mathrm{Au}$ niveau de la mise en œuvre, les politiques publiques sont confrontées à des difficultés issues de l'ineffectivité de l'autonomie institutionnelle et financière de la mairie.

\section{References:}

1. Adiko. (2003). La décentralisation en Côte d'Ivoire depuis l'ère colonial à nos jours. In Africa Research Séries: Vol. 10. L'administration locale en Côte D'Ivoire (p. 5-14). Consulté à l'adresse http://www.ide.go.jp/English/Publish/Download/Ars/10.html

2. Adomon, A. A. (2015). Bilan de la politique de décentralisation en Côte d'Ivoire: Cas des communes d'Alépé, de Taabo et de Yopougon (Doctorat en Géographie). Felix Houphouët Boigny, Cocody.

3. Anderson C. W. (1988). "Political jugement and theory in policy analysis ». In Handbook of Political Theory and Policy Science. New York: Portis E. B., Levy M. B., (dir.),.

4. Anderson, J. E. (1975). Public policy-making. Praeger.

5. Assepo, A. H.-. (2002). Développement, aménagement, régionalisation en Côte d'Ivoire. Presses Universitaires de Cote d'Ivoire.

6. Atta, K. (2001). Décentralisation et développement local. Gres IGT.

7. Atta, K., \& Gobe, T. (2013). Décentralisation et crise économique en Côte d'Ivoire. European Scientific Journal, 9.

8. Balme, R., \& Brouard, S. (2005). Les conséquences des choix politiques: Choix rationnel et action publique. Revue française de science politique, 55(1), 33-50.

9. Bede, C. (2017). Subventions dérisoires, budgets non approuvés : Les élus locaux interpellent le gouvernement. Notre voie.

10. Blundo, G. (2001). Négocier l'État au quotidien: Agents d'affaires, courtiers et rabatteurs dans les interstices de l'administration sénégalaise. Autrepart, $\quad$ (20), https://doi.org/10.3917/autr.020.0075

11. Caillosse, J. (1988). Le savoir juridique à l'épreuve de la décentralisation? Annuaire des collectivités locales, 8(1), 5-31. https://doi.org/10.3406/coloc.1988.1034

12. Chabanet, D., \& Guigni, M. (s. d.). Les conséquences des mouvements sociaux. In Penser les mouvements sociaux: Conflits sociaux et 
contestation dans les sociétés comptemporaines (p. 145-161). Paris : La découverte.

13. Degni Segui René. (2013). Droit administratif général (Vol. 1). Omniprésence.

14. Dégni-Ségui, R. (2013). Droit administratif général (4éd., Vol. 1). Omniprésence.

15. Douillet, A., \& Robert, C. (2007). Les élus dans la fabrique de l'action publique locale. Science de la société, (71), 2-24.

16. Downs, A. (2013). Une théorie économique de la démocratie. Bruxelles ; Paris: Université de Bruxelles.

17. Dramé, H. (1998). Décentralisation et Enjeux Politiques. L'Exemple du Conflit Casamançais (Sénégal). Bulletin de l'APAD, (16). Consulté à l'adresse http://journals.openedition.org/apad/538

18. Dupuy, C., \& Halpern, C. (s. d.). Les politiques publiques face à leurs protestataires. Revue française de science politique, 4(55), 701-722.

19. Éducation/Tiassalé : Le maire met fin aux cotisations Coges | FratMat. (s. d.). Consulté 2 novembre 2020, à l'adresse https://www.fratmat.info/article/208102/Soci\%C3\%A9t\%C3\%A9/\% C3\%89ducation/educationtiassale--le-maire-met-fin-aux-cotisationscoges

20. Gacha, T. (2009). Essai sur un état africain décentralisé : Le cas de la Côte d'Ivoire: contribution à l'étude du nouvel état africain (Thesis, Toulouse 1). Consulté à l'adresse http://www.theses.fr/2009TOU10049

21. Gerstle, J. (s. d.). La réactivité aux préférences collectives et l'imputabilité de l'action publique. Revue française de science politique, 6(53), 859-885.

22. Greffe, X. (2005). La décentralisation (Nouv. éd. ent. ref. et mise à jour). Paris: La Découverte.

23. Hassenteufel, P. (2010). Les processus de mise sur agenda : Sélection et construction des problèmes publics. Informations sociales, $n^{\circ}$ 157(1), 50-58.

24. Hassenteufel, P. (2011). Sociologie politique : L'action publique (2e édition). Armand Colin.

25. Hugon, P., Vimard, P., \& Tapinos, G. P. (2003). La Côte d'Ivoire à l'aube du XXIe siècle: Défis démographiques et Développement durable. Paris : Karthala.

26. Jean, P. (2013). Gestion des Coges : L'implication des élus locaux, une exigence. nouveau réveil.

27. Kübler, D., \& Maillard, J. de. (2009). Analyser les politiques publiques. Grenoble: Presses universitaires de Grenoble.

28. Lanoue, E. (2004). La société ivoirienne au fil de ses réformes 
scolaires: Une politique d'éducation « intermédiaire» est-elle possible? Autre part, (31).

29. Lascoumes, P., \& Galès, P. L. (2018). Sociologie de l'action publique-2e éd. (3e édition). Malakoff : Armand Colin.

30. Lugaz, C., De Grauwe, A., Balde, D., Diakhaté, C., Dougnon, D., Moustapha, M., \& Odushina, D. (s. d.). École et décentralisation: Résultats d'une recherche en Afrique francophone de l'Ouest. Paris : Institut international de planification de l'éducation.

31. Maillard, J. (s. d.). La conduite des politiques publiques à l'épreuve des temporalités électorales. Quelques hypothèses exploratoires. Pôle Sud, (25), 39-53.

32. Mambo, P. (2009). Droit et ville en Afrique: Étude de la décentralisation ivoirienne en matière d'urbanisme. Paris : Harmattan.

33. Marie, J., \& Idelman, E. (2010). La décentralisation en Afrique de l'Ouest: Une révolution dans les gouvernances locales? EchoGéo, (13). https://doi.org/10.4000/echogeo.12001

34. Mel, T. (2015). La décentralisation à l'épreuve de la crise économique en Côte d'Ivoire. Paris: harmattan.

35. Ministère de l'Administration du Territoire a organisé les, \& États Généraux de la Décentralisation et du Développement Local. (2007). Les actes des états généraux de la Décentralisation et du Développement Local.

36. Ministère de l'éducation nationale de la République de Côte d'Ivoire. (2016). Le financement de l'éducation en Côte d'Ivoire, 2006-2015 .Sur le modèle des Comptes Nationaux de l'Éducation Volume I : Analyse et annexe 1.

37. Mons, N. (2004). Politiques de décentralisation en éducation: Diversité internationale, légitimations théoriques et justifications empiriques. Revue française de pédagogie, 146(1), 41-52. https://doi.org/10.3406/rfp.2004.3092

38. Nahi, P. C. (2018). Les logiques autoritaires de la production de l'ordre en Côte d'Ivoire: De la crise de légitimité politique à la criminalisation des mouvements sociaux. Lonniya, 1(5).

39. N'tchuvi, T. Y.-C., \& Aloko, N. J. (2018). Analyse de La contribution Des collectivités territoriales au développement local En Côte D'ivoire: Cas du département d'agboville. European Scientific Journal, 14(32).

40. Ovono, E. A. (2012). L'autonomie financière des collectivités locales en Afrique noire francophone. Le cas du Cameroun, de la Côte d'Ivoire, du Gabon et du Sénégal. afrilex.

41. Palier, B., \& Surel, Y. (2005). Les « trois I» et l'analyse de l'État en action. Revue française de science politique, 55(1), 7-32. 
42. Perret, B. (2014). L'évaluation des politiques publiques (Nouvelle édition). Consulté à l'adresse https://www.lgdj.fr/l-evaluation-despolitiques-publiques-9782707183385.html

43. Pomey, M.-P., Morgan, S., Church, J., Forest, P.-G., Lavis, J. N., McIntosh, T., ... Dobson, S. (2010). Do provincial drug benefit initiatives create an effective policy lab? The evidence from Canada. Journal of Health Politics, Policy and Law, 35(5), 705-742. https://doi.org/10.1215/03616878-2010-025

44. Proteau, L. (2002). Passions scolaires en Côte d'Ivoire : École, état et société. Karthala.

45. Prud'homme, R. (1995). "Les dangers de la décentralisation (anglais). Observateur de Recherche à La Banque Mondiale, 10(2), 201-220.

46. Kei, M. (2010). Caractérisation et attitudes à l'égard des comités de gestion des établissements secondaires publics du district d'Abidjan. Revue Africaine d'Anthropologie, Nyansa-Pô, 10.

47. Rey, O. (2013). Décentralisation et politiques éducatives. Dossier d'actualité veille et analyses, (83).

48. Semiti, A. J. (2006). La programmation ou la planification implicite de l'échec scolaire au niveau du primaire et du secondaire en Côte d'ivoire pendant et apres la colonisation. Revue du CAMES - Nouvelle Série B, 007(1).

49. Soumahoro, M. (2015). Construction, reconstruction territoriale et décentralisation en Côte d'Ivoire (2002-2009). Espaces et sociétés, $n^{\circ}$ 160-161(1), 51-66.

50. Zadi, A. (2020). Suppression des cotisations du Coges : La Fesci donne un ultimatum à la ministre Camara Kandia. Infodrome.

51. Wodie, F. (1996). Institutions politiques et droit constitutionnel en Côte d'Ivoire. Presses universitaires de Côte d'Ivoire. 